\title{
COHERENT MODE OF A HIGH INTENSITY BEAM IN A SYNCHROTRON
}

\author{
$\underline{\text { S. Machida }}{ }^{1}$, T. Uesugi, KEK-Tanashi, Tanashi, Tokyo, 188-8501, Japan \\ M. Ikegami, JAERI, Tokai, Ibaraki, 319-1195, Japan
}

\begin{abstract}
As one of major sources which cause emittance growth and beam loss in high intensity hadron synchrotrons, we have investigated coherent mode oscillations in a beam. Using Sacherer's envelope equations, mode frequencies of a bunched beam are derived. Multi-particle simulation shows that emittance growth occurs when the oscillation frequency of a coherent mode hits a resonance. The present status of a beam experiment at HIMAC is also mentioned, which is expected to show the direct observation of a resonance crossing of a coherent mode and beam loss.
\end{abstract}

\section{INTRODUCTION}

Emittance growth and beam loss in high intensity accelerator draw more and more attention recently because of several projects, such as JHF, SNS, ESS, where high intensity accelerators are the essential components and their radioactivation has to be minimized. Study of a parametric resonance in linear accelerator has been successful for last few years [1]. In which, motion of particles in the tail of a beam core is treated in semi selfconsistent way, so-called particle-core model. In fact, similar mechanism may occur not only in linear accelerators but also in circular machines [2,3].

Stability of a coherent mode itself, not only as a driving force of halo formation, is one of major topics in a circular machine. In particular, an envelope mode and its stability in connection with resonances excited by field errors has been studied for long time based on envelope equations.

The envelope stability analysis can be found in his dissertation work of Sacherer [4]. He clearly stated that incoherent motion is not the one which causes beam loss. Using envelope equations, he showed that when a coherent mode frequency hits a resonance, the envelope becomes unstable and cause emittance growth and beam loss.

Although Sacherer's analysis holds in general, the real situation is rather complicated in a circular machine. First, there is an effect of dispersion functions in a circular machine. Much work has been done and shows that essentially the same form of envelope equations can be applicable to dispersion [5].

Secondly, a beam in an accelerator is bunched in 3D configuration space unlike the model assumed by Sacherer in his dissertation. In fact, the mode frequency is derived for an azimuthally symmetric 3D beam by Barnard et. al.
[6] and for a bunched beam in a linac with some approximations by Pabst et. al. [7]. However, for long bunch in a synchrotron, three mode frequencies are different from those results as we show later.

The ultimate confirmation of the model should be done by beam experiments. Although there are some experiments which detect coherent quadrupole mode, its connection with emittance growth and beam loss is still not clear.

In this paper, we first discuss envelope equations of a $2 \mathrm{D}$ and $3 \mathrm{D}$ beam. In order to estimate $3 \mathrm{D}$ potential due to a beam, elliptical integral is numerically estimated. Then, we quickly introduce a beam experiment at HIMAC. Finally, we will show some simulation results to support the model.

\section{COHERENT MODES}

As shown by Sacherer [8], 2D envelope equations for any particle distribution can be written in a self-consistent way if one takes rms beam size and emittance evolution is known.

$\frac{d^{2} \tilde{x}}{d \phi^{2}}+\left(v_{0 x}^{2}-\frac{N_{2} r_{0} R^{2}}{\beta^{2} \gamma^{3}} \frac{1}{\tilde{x}(\tilde{x}+\tilde{y})}\right) \tilde{x}=\frac{\left(R \varepsilon_{x}\right)^{2}}{\tilde{x}^{3}}$

where $N_{2}$ is the line density of particles, $r_{0}$ the classical proton radius, $R$ the average radius of a machine, $\beta$ and $\gamma$ Lorentz factors. Similar equation for $y$. When the bare tune of two planes are split, two equations are practically independent and two mode frequencies are mostly determined by two bare tunes and tune shift of each plane, respectively.

On the other hand, when the two bare tunes are nearly equal, the two modes are coupled. One calls the higher mode as breathing mode and the lower one as quadrupole mode because the former mode oscillates in phase and the latter does out of phase in two planes.

The envelope equations are easily extended to include longitudinal mode. In fact, Sacherer showed that once one takes rms beam size, the envelope equations for a bunched beam also hold almost independently of the detailed particle distribution just like a 2D beam [8]. However, analytically treatment becomes difficult since the force term remains as elliptical integral. The 3D envelope equations are

$\frac{d^{2} \tilde{x}}{d \phi^{2}}+\left(v_{0 x}^{2}-\frac{N_{3} r_{0} R^{2}}{\beta^{2} \gamma^{3}} \lambda_{3} g(\tilde{x}, \tilde{y}, \tilde{z})\right) \tilde{x}=\frac{\left(R \varepsilon_{x}\right)^{2}}{\tilde{x}^{3}}$

\footnotetext{
${ }^{1}$ E-mail address: shinji.machida@kek.jp
} 
where $N_{3}$ is the total number of particles, $\lambda_{3}$ a factor defined by Sacherer indicating small difference among distributions, and $g$ elliptical integral as shown below. Similar equations for $y$ and $z$.

$$
g(a, b, c)=\frac{3}{2} \int_{0}^{\infty} \frac{d s}{\left(a^{2}+s\right)^{3 / 2}\left(b^{2}+s\right)^{1 / 2}\left(c^{2}+s\right)^{1 / 2}}
$$

We numerically integrate elliptical integral and derive three mode frequencies and eigen vectors [9]. As a result, we can show that in a long bunch such as the one in a synchrotron, the two transverse modes of a bunched beam are almost identical to the ones of a $2 \mathrm{D}$ beam. That approximation holds unless the longitudinal bare tune becomes similar to the transverse one, which never occurs in synchrotrons. Third mode presents longitudinal oscillations.

\section{EXPERIMENT AT HIMAC}

Now we know that the difference of transverse mode frequencies between a 2D and 3D beam are negligible in a synchrotron. In order to verify the Sacherer's model and study mechanism of emittance growth and beam loss, or halo formation due to core oscillations, we started a beam experiment in HIMAC.

HIMAC is a heavy ion synchrotron and we use $\mathrm{He}^{2+}$ beams because the tune shift becomes largest. Main machine parameters are shown in Table 1.

Table 1: Main machine parameters of HIMAC.

\begin{tabular}{|l|c|c|}
\hline \multicolumn{1}{|c|}{ item } & number & units \\
\hline Energy & 6 & $\mathrm{MeV} / \mathrm{u}$ \\
\hline Intensity & $110^{11}$ & $\mathrm{ppp}$ \\
\hline Particle & $\mathrm{He}^{2+}$ & \\
\hline Emittance & $264(\mathrm{H}) / 10(\mathrm{~V})$ & $\pi \mathrm{mm}-\mathrm{mrad}$ \\
\hline
\end{tabular}

A pickup with four electrodes was designed and installed. It was expected to detect coherent signal of a quadrupole mode. An RF quadrupole kicker is also used to excite a quadrupole mode in a beam.

Up to now, we have observed coherent signals. The quadrupole coherent tune shift is $-0.082 / 10^{11} \mathrm{ppp}$ while the expected value is $-0.059 / 10^{11} \mathrm{ppp}$. A part of the discrepancy can be attributed to uncertainty of emittance. The relation between beam loss and resonance of coherent mode is not clear yet. More detailed descriptions on the experiment are found in another paper [9].

\section{SIMULATION RESULTS}

A multi-particle tracking is the other way to verify the mode frequency with space charge force and also to see its relation to beam emittance and loss. In a conventional way of single particle tracking, space charge force is included as a momentum kick at every time step [10]. The time step is chosen as small enough such that space charge force is evaluated smoothly according to a beam envelope. That is necessary in order to estimate a frequency of asymmetric mode such as quadrupole one. As a model lattice, HIMAC is adopted, where about 380 space charge kicks are applied per turn while the beam envelope oscillates 12 times (essentially 12 FODO cells in a whole ring.)

First, we look at oscillation frequencies of second moments in a $2 \mathrm{D}$ beam without space charge. The vertical bare tune is lowered as a function of time by decreasing strength of defocusing quadrupoles. The initial bare tune is $(3.65,3.60)$ and the vertical one is varied at the rate of 0.10 per 2000 turns. Figure 1 shows four modes of coherent motion of second moments, which correspond to $2 v_{x}, 2 v_{y}, v_{x}+v_{y}$, and $v_{x}-v_{y}$.

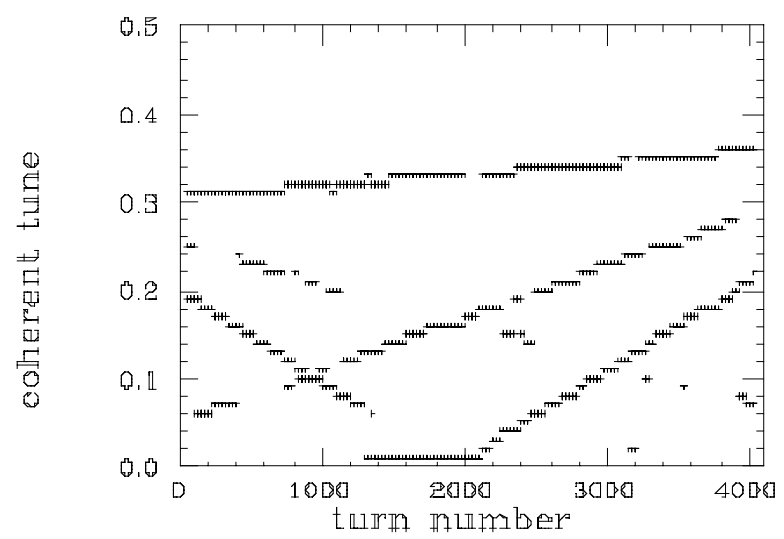

Figure 1: Tune of coherent modes of second moments. Space charge force is not included.

The line starting from 0.3 corresponds to $2 v_{x}(=23.65-$ 7 ), which is slightly increasing because of defocusing quadrupole change. The one starting from 0.2 and going down as the turn number increases is $2 v_{\mathrm{y}}(=23.60-7)$, which crosses zero and going down although the figure shows its reflection. The other two lines starting from 0.25 and 0.05 show $v_{x}+v_{y}$, and $v_{x}-v_{y}$, respectively.

There are some quadrupole errors included in the model lattice. The half-integer resonance with a width of 0.005 is excited at $v_{y}=3.5$. Figure 2 shows rms emittance under the same condition of time-varying quadrupole as Fig. 1.

Initial values of horizontal and vertical rms emittance (normalized) are $7.310^{-6}$ and $2.810^{-7}$, respectively. When the vertical bare tune crosses a half-integer resonance at 3.5 (and also coherent tune becomes integer of 7), vertical rms emittance suddenly grows. The decrease of emittance right after the crossing is due to beam loss (once a particle satisfies loss criterion, that particle is no longer counted to calculate emittance.) 


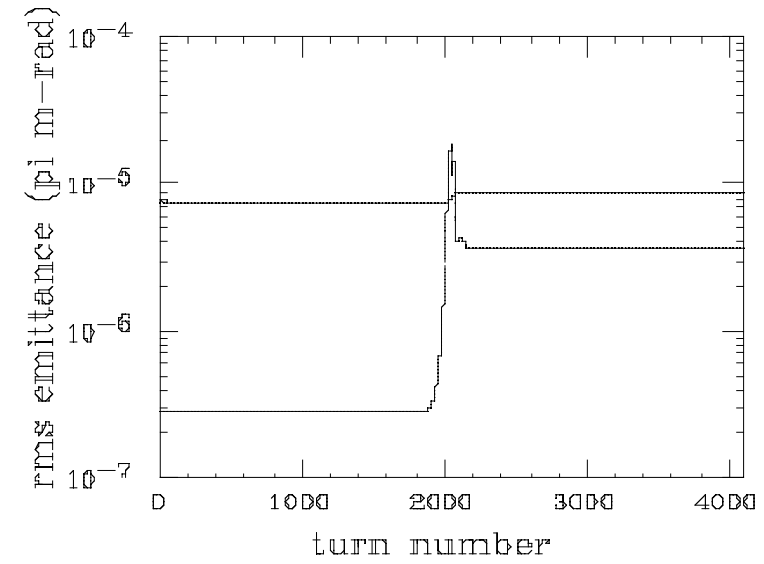

Figure 2: Horizontal and vertical rms emittance when the bare tune is varied in the same way of Fig. 1.

Now, we include space charge effects. We first examine $\mathrm{KV}$ distribution in which space charge force is linear. The beam intensity was chosen to be $110^{11} \mathrm{ppp}$, which makes the vertical incoherent tune shift of -0.06 (much smaller shift in horizontal because of its larger emittance.)

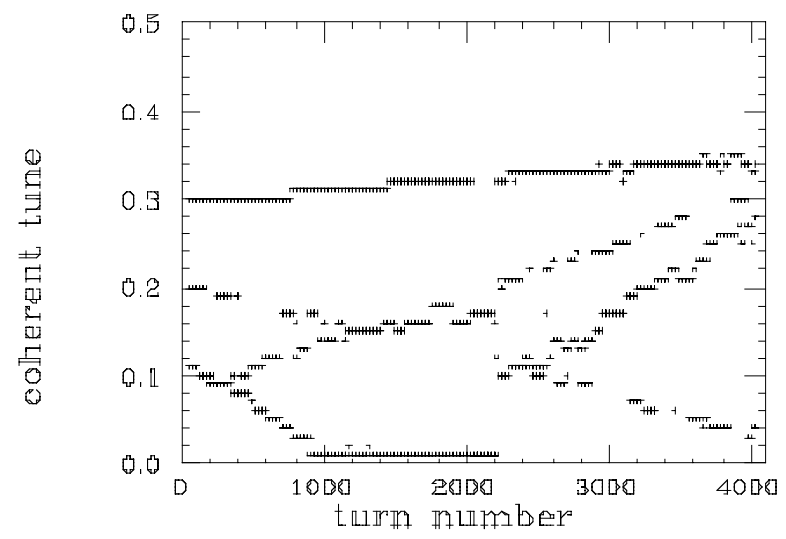

Figure 3: Tune of coherent modes with space charge force.

Figure 3 shows same four modes of Fig. 1. Because of space charge defocusing force, the coherent tune which is determined by vertical motion is lowered. The lowered coherent tune is no longer twice of loaded incoherent tune as the envelope equations states. At the turn number of around 1200 , the coherent mode of $2 v_{\mathrm{y}}$ becomes zero. If one looks at rms emittance, one can notice that the rms emittance starts increasing right after coherent tune crosses integer (in this case 7.) The growth continues up to the turn number of 2000, where the bare tune hits the resonance.

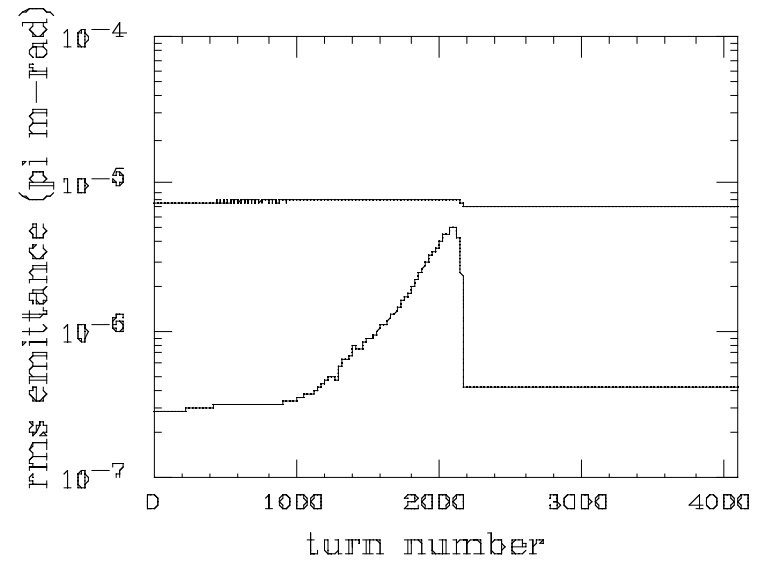

Figure 4: Horizontal and vertical rms emittance when the vertical bare tune is lowered.

That behavior is weakly dependent of particle distribution. The waterbag and gaussian distributions give almost same results.

Finally, let me mention the simulation results of a 3D beam. Since long term ( 1,000 turns) tracking with space charge is very time consuming, we have not confirmed the emittance growth and beam loss due to a resonance crossing of a coherent mode yet. However, a mode frequency is identified. Under the condition of the same incoherent tune as Fig. 3 (without tune ramping), the coherent tune of $2 \mathrm{v}_{\mathrm{y}}$ becomes 0.12 . That is in good agreement with our previous findings, namely $2 \mathrm{D}$ and $3 \mathrm{D}$ mode frequencies are similar.

\section{REFERENCES}

[1] See, for example, T. P. Wangler, et. al., "Particlecore model for transverse dynamics of beam halo", Phys. Rev. ST-AB, 1, 1998.

[2] S. Machida, "Coherent mode analysis of high intensity beams in synchrotrons", Proc. of 1998.

[3] J. A. Holmes, et. al., "A particle core model for space charge dynamics in rings", ibid.

[4] F. J. Sacherer, "Transverse space-charge effects in circular accelerators", Ph.D, Thesis, UCRL-18454, October, 1968.

[5] S. Y. Lee and H. Okamoto, "Space-charge dominated beams in synchrotrons", Phys. Rev. Lett. 80, 1998.

[6] J. J. Barnard, et. al., "Theory of longitudinal beam halo in RF linacs: I. Core/test-particle formation", Proc. of 1997 PAC.

[7] M. Pabst, et. al., "Progress on intense proton beam dynamics and halo formation", Proc. of 1998 EPAC.

[8] F. J. Sacherer, "RMS envelope equations with space charge", IEEE trans. Nucl. Phys., 1991.

[9] T. Uesugi, et. al., in this proceedings.

[10] S. Machida, "Simulation of space charge effects in a synchrotron", AIP Conf. Proc. 448, 1998. 